\title{
Stability Conditions for Perturbed Semigroups on a Hilbert Space via Commutators
}

\author{
Michael Gil' 1*
}

\author{
Abstract \\ Let $A$ and $B$ be linear operators on a Hilbert space. Let $A$ and $A+B$ generate $C_{0}$-semigroups $e^{t A}$ and $e^{t(A+B)}$, \\ respectively, and $e^{t A}$ be exponentially stable. We establish exponential stability conditions for $e^{t(A+B)}$ in terms of \\ the commutator $A B-B A$, assuming that it has a bounded extension. Besides, $B$ can be unbounded. \\ Keywords: Commutator, Hilbert space, Semigroups, Stability \\ 2010 AMS: Primary 47D06, Secondary 47D60
}

${ }^{1}$ Department of Mathematics, Ben Gurion University of the Negev, Beer-Sheva 84105, Israel, ORCID: orcid.org/0000-0002-6404-9618

*Corresponding author: gilmi@bezeqint.net

Received: 4 January 2019, Accepted: 24 April 2019, Available online: 27 June 2019

\section{Statement of the main result}

Let $\mathscr{H}$ be a Hilbert space with a scalar product $\langle.,$.$\rangle , the norm \|\|=.\sqrt{\langle, .\rangle}$ and unit operator $I$. For a linear operator $C$, Dom $(C)$ is the domain, $C^{*}$ is the adjoint operator, $\sigma(C)$ is the spectrum. If $C$ is a bounded operator, then $\|C\|$ is its operator norm.

Throughout this paper $A$ and $B$ are linear operators on $\mathscr{H}$ with $\operatorname{Dom}(B) \supseteq \operatorname{Dom}(A)$. In addition, $A$ and $A+B$ generate $C_{0}$-semigroups $e^{A t}$ and $e^{t(A+B)}$, respectively.

We consider the following problem: let $e^{A t}$ be exponentially stable, i.e.

$$
\left\|e^{A t}\right\| \leq c e^{-v t}(t \geq 0 ; c=\text { const } \geq 1, v=\text { const }>0)
$$

What are the conditions that provide the exponential stability of $e^{t(A+B)}$ ? The literature on the stability of semigroups is very rich. The classical results are presented in the books [1, 2], about the recent investigations for instance see [3] -[6], $[7,8,9,10]$. In particular, in [7] the author investigates the uniform, strong, weak and almost weak stabilities of multiplication semigroups on Banach space valued $L^{p}$-spaces. In the paper [9] Lyapunov based proofs are presented for the well-known Arendt-Batty-Lyubich-Vu Theorem for strongly continuous and discrete semigroups. In [10] the authors obtain continuous-time and discrete-time Lyapunov operator inequalities for the exponential stability of strongly continuous, one-parameter semigroups acting on Banach spaces. Thus they extend the classic result of Datko from Hilbert spaces to Banach spaces. Recall also that various conditions, under which the perturbed operator generates a $C_{0}$-semigroup can be found for instance in [11, Chapter III]. For example, if $B$ is $A$-compact and the semigroups generated by $A$ is analytic, then by Corollary III.2.17 from [11, p. 180] $A+B$ generates an analytic semigroup. Certainly, we could not survey the whole subject here and refer the reader to the above listed publications and references given therein.

To the best of our knowledge, the exponential stability conditions for the perturbed semigroup in terms of the commutator $[A, B]=A B-B A$ have not been investigated in the available literature. In the paper [12] in the case of a Banach space, an 
estimate has been established for the $L^{1}$-norm of a semigroup generated by $A+B$, provided that both $[A, B]$ and $B$ are bounded. The aim of this paper is to establish exponential stability conditions for $e^{t(A+B)}$ in terms of $[A, B]$, assuming that

$$
B \operatorname{Dom}\left(A^{2}\right) \subseteq \operatorname{Dom}(A)
$$

and

$[A, B]$ has a bounded extension.

Besides, $B$ can be unbounded. Since $A$ generates a $C_{0}$-semigroup, $\operatorname{Dom}\left(A^{2}\right)$ is dense in $\mathscr{H}$, cf. [13, Theorem I.2.3]. So the operators $A B$ and $B A$ are defined on $\operatorname{Dom}\left(A^{2}\right)$. Thus (1.2) means that $[A, B]$ is defined and uniformly bounded on $\operatorname{Dom}\left(A^{2}\right)$, and therefore admits the extension to the whole space as a bounded operator. Our approach in the present paper is considerably different from the one in [12]. In addition, we considerably generalize the main result from [14].

Introduce the operator

$$
W:=\int_{0}^{\infty} e^{A^{*} t} e^{A t} d t
$$

This integral converges in the operator norm, since $e^{A t}$ is exponentially stable, and

$$
\|W\| \leq \int_{0}^{\infty}\left\|e^{A t}\right\|^{2} d t \leq c^{2} \int_{0}^{\infty} e^{-2 v t} d t=\frac{c^{2}}{2 v} .
$$

The integral

$$
\zeta(A):=2 \int_{0}^{\infty}\left\|e^{A t}\right\| \int_{0}^{t}\left\|e^{s A}\right\|\left\|e^{(t-s) A}\right\| d s d t
$$

also converges, and

$$
\zeta(A) \leq 2 c^{3} \int_{0}^{\infty} e^{-v t} \int_{0}^{t} e^{-v s} e^{-v(t-s)} d s d t=2 c^{3} \int_{0}^{\infty} e^{-2 v t} t d t=\frac{c^{3}}{2 v^{2}} .
$$

Finally assume that

$$
\Lambda(B):=\sup _{h \in \operatorname{Dom}(B) ;\|h\|=1} \Re\langle B h, h\rangle<\infty
$$

and put

$$
\psi(W, B):= \begin{cases}2 \Lambda(B)\|W\| & \text { if } \Lambda(B)>0 \\ 0 & \text { if } \Lambda(B) \leq 0 .\end{cases}
$$

Now we are in a position to formulate the main result of the paper.

Theorem 1.1. Let conditions (1.1) and (1.2) hold, and $e^{A t}$ be exponentially stable. If, in addition, $\Lambda(B)<\infty$ and

$$
\psi(W, B)+\|[A, B]\| \zeta(A)<1,
$$

then $e^{t(A+B)}$ is also exponentially stable.

This theorem is proved in the next section. It is sharp. Indeed, let $A$ and $B$ be commuting normal operators, with $\alpha(A):=\sup \Re \sigma(A)<0 . \Lambda(B)=\alpha(B)>0$. Then $\left\|e^{A t}\right\|=e^{\alpha(A) t}(t \geq 0)$, and by (1.3) $\|W\| \leq \frac{1}{2|\alpha(A)|}$. Consequently,

$$
\psi(W, B)=\frac{\alpha(B)}{|\alpha(A)|}
$$

By Theorem 1.1 $e^{t(A+B)}$ is stable if $\alpha(B)<|\alpha(A)|$. But $\left\|e^{t(A+B)}\right\|=e^{(\alpha(A)+\alpha(B)) t}(t \geq 0)$. Therefore, in the considered case $e^{t(A+B)}$ is stable, provided $\alpha(A)+\alpha(B)<0$. So Theorem 1.1 is really sharp. 


\section{Proof of Theorem 1.1}

Lemma 2.1. Let $A$ generate a $C_{0}$-semigroup $e^{A t}$ on $\mathscr{H}$, and conditions (1.1) and (1.2) hold. Then the operator $\left[e^{A t}, B\right]:=$ $e^{A t} B-B e^{A t}$ is bounded. Moreover,

$$
\left[e^{A t}, B\right]=\int_{0}^{t} e^{s A}[A, B] e^{(t-s) A} d s(t \geq 0) .
$$

Proof. For any $x \in \operatorname{Dom}\left(A^{2}\right)$, we have $e^{s A} x \in \operatorname{Dom}\left(A^{2}\right)$ and $A e^{s A} x \in \operatorname{Dom}(A) \subseteq \operatorname{Dom}(B)$. So $B A e^{s A} x \in \mathscr{H}$. In addition, according to (1.1), $A B e^{s A} x \in \mathscr{H}$. Thus,

$$
e^{A(t-s)}(A B-B A) e^{s A} x \in \mathscr{H}\left(x \in \operatorname{Dom}\left(A^{2}\right)\right)
$$

But

$$
e^{A(t-s)}(A B-B A) e^{s A} x=-\frac{\partial}{\partial s} e^{A(t-s)} B x-B \frac{\partial}{\partial s} e^{s A} x=-\frac{\partial}{\partial s} e^{A(t-s)} B e^{s A} x .
$$

Integrating this equality, we get

$$
\begin{aligned}
\int_{0}^{t} e^{A(t-s)}(A B-B A) e^{s A} x d s & =-\int_{0}^{t} \frac{\partial}{\partial s} e^{A(t-s)} B e^{s A} x d s=-\left.e^{A(t-s)} B e^{s A} x\right|_{0} ^{t} \\
& =\left(e^{t A} B-B e^{t A}\right) x
\end{aligned}
$$

Thus

$$
\left[e^{A t}, B\right] x=\int_{0}^{t} e^{A(t-s)}[A, B] e^{s A} x d s .
$$

Since $[A, B]$ is bounded, we can extend $\left[e^{A t}, B\right]$ to the whole space. This proves the required relation (2.1).

Proof of Theorem 1.1: Since $e^{A t}$ is exponentially stable, $W$ is a unique solution of the Lyapunov equation

$$
W A+(W A)^{*}=-I .
$$

Equation (2.2) is understood in the sense

$$
\left\langle A z_{1}, W z_{2}\right\rangle+\left\langle W z_{1}, A z_{2}\right\rangle=-\left\langle z_{1}, z_{2}\right\rangle\left(z_{1}, z_{2} \in \operatorname{Dom}(A)\right) .
$$

Besides, $W: \operatorname{Dom}(A) \rightarrow \operatorname{Dom}\left(A^{*}\right)$, cf. [1, p. 252, Section 5.3].

For all $h \in \operatorname{Dom}(A)$ with $\|h\|=1$, by (2.3) we can write

$$
\begin{aligned}
\langle(A+B) h, W h\rangle+\langle W h,(A+B) h\rangle & =\langle A h, W h\rangle+\langle W h, A h\rangle+\langle B h, W h\rangle+\langle W h, B h\rangle \\
& =-1+\langle B h, W h\rangle+\langle W h, B h\rangle=-1+\left\langle B h, \int_{0}^{\infty} e^{A^{*} t} e^{A t} d t h\right\rangle+\left\langle\int_{0}^{\infty} e^{A^{*} t} e^{A t} d t h, B h\right\rangle \\
& =-1+\int_{0}^{\infty}\left(\left\langle e^{A t} B h, e^{A t} h\right\rangle+\left\langle e^{A t} h, e^{A t} B h\right\rangle\right) d t=-1+\int_{0}^{\infty}\left(\left\langle B e^{A t} h, e^{A t} h\right\rangle+\left\langle e^{A t} h, B e^{A t} h\right\rangle\right) d t \\
& +\int_{0}^{\infty}\left(\left\langle\left[e^{A t}, B\right] h, e^{A t} h\right\rangle+\left\langle e^{A t} h,\left[e^{A t}, B\right] h\right\rangle\right) d t .
\end{aligned}
$$

Thus,

$$
\langle(A+B) h, W h\rangle+\langle W h,(A+B) h\rangle=-1+J_{1}(h)+J_{2}(h),
$$

where

$$
J_{1}(h)=\int_{0}^{\infty}\left(\left\langle B e^{A t} h, e^{A t} h\right\rangle+\left\langle e^{A t} h, B e^{A t} h\right\rangle\right) d t
$$

and

$$
J_{2}(h)=\int_{0}^{\infty}\left(\left\langle\left[e^{A t}, B\right] h, e^{A t} h\right\rangle+\left\langle e^{A t} h,\left[e^{A t}, B\right] h\right\rangle\right) d t .
$$


Since

$$
\left\langle B e^{A t} h, e^{A t} h\right\rangle+\left\langle e^{A t} h, B e^{A t} h\right\rangle=2 \Re\left\langle B e^{A t} h, e^{A t} h\right\rangle \leq 2 \Lambda(B)\left\langle e^{A t} h, e^{A t} h\right\rangle,
$$

we have

$$
J_{1}(h) \leq 2 \Lambda(B) \int_{0}^{\infty}\left\langle e^{A t} h, e^{A t} h\right\rangle d t=2 \Lambda(B)\langle W h, h\rangle .
$$

If $\Lambda(B)>0$, then $J_{1}(h) \leq 2 \Lambda(B)\|W\|$. If $\Lambda(B)<0$, then $J_{1}(h) \leq 0$. So $J_{1}(h) \leq \psi(W, B)$. In addition, by Lemma 2.1

$$
\left|J_{2}(h)\right| \leq 2 \int_{0}^{\infty}\left\|e^{A t}\right\|\left\|\left[e^{A t}, B\right]\right\| d t \leq 2 \int_{0}^{\infty}\left\|e^{A t}\right\|\|[A, B]\| \int_{0}^{t}\left\|e^{s A}\right\|\left\|e^{(t-s) A}\right\| d s d t=\|[A, B]\| \zeta(A) .
$$

Consequently, due to (1.5), for all $h \in \operatorname{Dom}(A),\|h\|=1$,

$$
\langle(A+B) h, W h\rangle+\langle W h,(A+B) h\rangle=-1+J_{1}(h)+J_{2}(h) \leq-(1-\psi(W, B)-\|[A, B]\| \zeta(A))<0 .
$$

Now the required result is due to the generalized Lyapunov theorem [2, Theorem 7.1].

\section{Example}

Let $\mathscr{H}=L^{2}(0,1)$, where $L^{2}(0,1)$ is the space of square-integrable functions defined on $[0,1]$ with the traditional scalar product. Let $a(x)$ be a complex valued function having a bounded measurable derivative, $b$ be a real constant,

$$
(A f)(x)=\frac{d^{2} f(x)}{d x^{2}}+a(x) f(x) \text { and }(B f)(x)=b f^{\prime}(x)(0<x<1, f \in \operatorname{Dom}(A))
$$

with

$$
\operatorname{Dom}(A)=\left\{h \in L^{2}(0,1): h^{\prime \prime} \in L^{2}(0,1), h(0)=h(1)=0\right\} .
$$

Then the commutator is defined by $([A, B] f)(x)=-b a^{\prime}(x) f(x)$ and $\|[A, B]\|=|b| \sup _{x}\left|a^{\prime}(x)\right|$. Clearly $A+B$ and $A$ generate $C_{0}$-semigroups. Assume that $q:=\max _{x} \Re a(x)<\pi^{2}$. Since the largest eigenvalue of the operator defined on $\operatorname{Dom}(A)$ by $d^{2} / d x^{2}$ is $-\pi^{2}$, we easily obtain

$$
\sup _{h \in \operatorname{Dom} A ;\|h\|=1} \Re\langle A h, h\rangle \leq q-\pi^{2}<0 .
$$

So $A$ is dissipative and therefore,

$$
\left\|e^{A t}\right\| \leq \exp \left[-t\left(\pi^{2}-q\right)\right](t \geq 0) .
$$

Hence, by (1.4)

$$
\zeta(A) \leq \frac{1}{2\left(\pi^{2}-q\right)^{2}}
$$

Since $\left(f^{\prime}, f\right)=-\left(f, f^{\prime}\right)(f \in \operatorname{Dom}(A))$, we have $\Lambda(B)=0$ and consequently, $\psi(W, B)=0$. Thus, due to Theorem 1.1 the semigroup generated by the operator $\tilde{A}=A+B$ defined by

$$
(\tilde{A} f)(x)=\frac{d^{2} f(x)}{d x^{2}}+b f^{\prime}(x)+a(x) f(x) \quad(0<x<1, f \in \operatorname{Dom}(A))
$$

is exponentially stable, provided

$$
|b| \sup _{x}\left|a^{\prime}(x)\right|<2\left(\pi^{2}-q\right)^{2} .
$$




\section{A particular case}

In this section we refine Theorem 1.1, assuming that

$$
\lambda(A):=\inf _{h \in \operatorname{Dom}(A) ;\|h\|=1} \Re\langle A h, h\rangle>-\infty .
$$

For example, let

$$
(A f)(x)=\frac{d f(x)}{d x}+a(x) f(x) \quad\left(0<x<1 ; f \in L^{2}(0,1)\right)
$$

with

$\operatorname{Dom}(A)=\left\{h \in L^{2}(0,1): h^{\prime} \in L^{2}(0,1), h(0)=h(1)\right\}$

and a complex bounded measurable function $a(x)$ with $\sup _{x} \Re a(x)<0$. Simple calculations show that in this case $\lambda(A)=$ $\inf _{x} \Re a(x)>-\infty$.

Furthermore, put

$$
\hat{\psi}(W, B):= \begin{cases}2 \Lambda(B)\|W\| & \text { if } \Lambda(B) \geq 0, \\ \frac{\Lambda(B)}{|\lambda(A)|} & \text { if } \Lambda(B)<0 .\end{cases}
$$

Theorem 4.1. Let conditions (1.1), (1.2) and (4.1) hold, and $e^{A t}$ be exponentially stable. If, in addition, $\lambda(B)<\infty$ and

$$
\hat{\psi}(W, B)+\|[A, B]\| \zeta(A)<1,
$$

then $e^{t(A+B)}$ is also exponentially stable.

Proof. Define $J_{1}(h)$ and $J_{2}(h)(h \in \operatorname{Dom}(A),\|h\|=1)$ as in Section 2. Under condition $\Lambda(B)<0$ we have

$$
J_{1}(h)=2 \int_{0}^{\infty} \Re\left\langle B e^{A t} h, e^{A t} h\right\rangle d t \leq 2 \Lambda(B) \int_{0}^{\infty}\left\langle e^{A t} h, e^{A t} h\right\rangle d t<0 .
$$

Put $y(t)=e^{A t} h(h \in \operatorname{Dom}(A))$. Then

$$
\frac{d}{d t}\langle y(t), y(t)\rangle=2 \Re\left\langle\frac{d}{d t} y, y(t)\right\rangle=2 \Re\langle A y, y(t)\rangle \geq 2 \lambda(A)\langle y(t), y(t)\rangle .
$$

Solving this inequality, we get

$$
\left\|e^{A t} h\right\| \geq e^{\lambda(A) t}\|h\| .
$$

Since $A$ generates a stable semigroup $\lambda(A)<0$. Consequently,

$$
\int_{0}^{\infty}\left\langle e^{A t} h, e^{A t} h\right\rangle d t \geq \int_{0}^{\infty} e^{2 \lambda(A)} d t\langle h, h\rangle=\frac{1}{2|\lambda(A)|}\langle h, h\rangle .
$$

If $\Lambda(B) \geq 0$, then according to (2.5) $\left|J_{1}(h)\right| \leq 2 \Lambda(B)\|W\|$. Thus $\left|J_{1}(h)\right| \leq \hat{\psi}(W, B)$. Taking into account (2.4) and (2.6), under condition (4.1) for all $h \in \operatorname{Dom}(A),\|h\|=1$, we obtain

$$
\langle(A+B) h, W h\rangle+\langle W h,(A+B) h\rangle=-1+J_{1}(h)+J_{2}(h) \leq-(1-\hat{\psi}(W, B)-\|[A, B]\| \zeta(A))<0 .
$$

Now the required result is due to the above mentioned generalized Lyapunov theorem [2, Theorem 7.1].

\section{Acknowledgement}

I am very grateful to the referee of this article for his (her) helpful remarks. 


\section{References}

[1] R. Curtain, H. Zwart, Introduction to Infinite-Dimensional Systems Theory, Springer, New York, 1995.

[2] T. Eisner, Stability of Operators and Operator Semigroups, Operator Theory: Advances and Applications, Vol. 209, Birkhăuser Verlag, Basel, 2010.

[3] A. Batkai, K.-J. Engel, J. Pruus, R. Schnaubelt, Polynomial stability of operator semigroups, Math. Nachr., 279 (13-14) (2006), 1425-1440.

[4] C.J.K. Batty, T. Duyckaerts, Non-uniform stability for bounded semi-groups on Banach spaces, J. Evol. Equ., 8 (2008), 765-780.

[5] C. Buse, A. Khan, G. Rahmat, O. Saierli, Weak real integral characterizations for exponential stability of semigroups in reflexive spaces, Semigr. Forum, 88 (2014), 195-204.

[6] C. Buse, C. Niculescu, A condition of uniform exponential stability for semigroups, Math. Inequal. Appl. 11(3) (2008), 529-536.

[7] R. Heymann, Eigenvalues and stability properties of multiplication operators and multiplication semigroups, Math. Nachr., 287(5-6) (2014), 574-584.

[8] L. Maniar, S.Nafiri, Approximation and uniform polynomial stability of $C_{0}$-semigroups, ESAIM Control Optim. Calc. Var., 22(1) (2016), 208-235.

[9] L. Paunonen, H. Zwart, A Lyapunov approach to strong stability of semigroups, Syst. \& Control Let., 62 (2013), $673-678$.

[10] C. Preda, P. Preda, Lyapunov operator inequalities for exponential stability of Banach space semigroups of operators, Appl. Math. Let., 25 (2012), 401-403.

[11] K.-J. Engel, R. Nagel, One-Parameter Semigroups for Linear Evolution Equations, Springer, New York, 2000.

[12] M.I. Gil', Semigroups of sums of two operators with small commutators, Semigroup Forum, 98(1) (2019), 22-30.

[13] S.G. Krein, Linear Equations in a Banach Space, Amer. Math. Soc., Providence, R.I, 1971.

[14] M.I. Gil', Stability of sums of operators, Ann. Univ. Ferrara, 62 (2016), 61-70. 